\title{
DIFERENÇAS NO CONSUMO DE ALIMENTOS ENTRE HOMENS E MULHERES ENTREVISTADOS PELO INQUÉRITO TELEFÔNICO VIGITEL
}

\author{
Differences in food consumption between men and women interviewed by VIGITEL telephone \\ survey
}

\section{Diferencias del consumo de alimentos entre hombres y mujeres entrevistados por la encuesta telefónica VIGITEL}

\section{Gabriela Dalcin Durante}

Universidade Federal de Mato Grosso - UFMT - Cuiabá (MT) - Brasil

\section{Lenir Vaz Guimarães}

Universidade Federal de Mato Grosso - UFMT - Cuiabá (MT) - Brasil

\section{Neuber José Segri}

Universidade Federal de Mato Grosso - UFMT - Cuiabá (MT) - Brasil

\author{
Maria Silvia Amicucci Soares Martins \\ Universidade Federal de Mato Grosso - UFMT - Cuiabá (MT) - Brasil
}

\section{Deborah Carvalho Malta}

Universidade Federal de Minas Gerais - UFMG - Belo Horizonte (MG) - Brasil

\section{RESUMO}

Objetivo: Analisar a frequência de consumo de alimentos marcadores de dieta saudável e não saudável entre homens e mulheres adultos e os fatores demográficos e socioeconômicos associados. Métodos: Estudo de corte transversal, de base populacional, com dados do Sistema de Vigilância de Fatores de Risco e Proteção para Doenças Crônicas não Transmissíveis por Inquérito Telefônico (VIGITEL). Foram incluídos 1.005 adultos ( 400 homens e 605 mulheres), de 20 a 59 anos, residentes em Cuiabá, Mato Grosso, entrevistados entre fevereiro e dezembro de 2014. As variáveis do estudo foram o consumo de alimentos marcadores de dieta saudável e não saudável e variáveis demográficas e socioeconômicas. Na análise de dados, utilizou-se análise bivariada por meio do teste de qui-quadrado (Rao-Scott) e teste de tendência linear. Resultados: A média de idade dos participantes foi de 37,9 anos para mulheres e 36,3 anos para os homens, dos quais 50,9\% (n=605) eram mulheres, $60,0 \%(n=455)$ eram adultos jovens, de 20 a 39 anos, e de raça/cor preta ou parda $(69,7 \% ; n=569)$. Maior consumo regular de frutas e hortaliças foi observado entre mulheres $(\mathrm{p}=0,01)$, entre aquelas de maior escolaridade e da raça/cor branca, e maior consumo de feijão foi observado entre os homens $(p=0,04)$. O consumo de carnes com excesso de gordura foi maior entre os homens $(p<0,01)$, assim como o consumo regular de doces $(\mathrm{p}=0,02)$ e a substituição das refeições principais por lanches foi maior entre as mulheres $(\mathrm{p}=0,01)$, ambos diretamente associados à escolaridade. Conclusão: Verificaram-se diferenças importantes no consumo de alimentos entre homens e mulheres residentes em Cuiabá, Mato Grosso. A escolaridade e a raça/cor foram variáveis que se associaram à maioria dos marcadores de consumo alimentar.

Descritores: Consumo de Alimentos; Inquéritos Epidemiológicos; Vigilância em Saúde Pública.

\section{ABSTRACT}

Objective: To analyze the frequency of consumption of healthy and unhealthy diet markers among adult men and women and the associated demographic and socioeconomic factors. Methods: Cross-sectional population-based study with data from the Telephone-based Surveillance of Risk and Protective Factors for Chronic Diseases (VIGITEL). A total of 1,005 adults (400 men and 605 women) aged 20 to 59 years living in Cuiabá, Mato Grosso, were interviewed between February and December 2014. The study variables were the consumption of healthy and unhealthy diet markers, and demographic and socioeconomic variables. Data underwent bivariate analysis using the chi-squared test (RaoScott) and linear trend test. Results: Participants' mean age was 37.9 years for women and 36.3 years for men. In all, 50.9\% ( $n=605)$ of the participants were women, $60.0 \%(n=455)$ were young adults aged 20 to 39 years, and black or parda ethnicity $(69.7 \%$; $n=569)$. Higher regular consumption of fruit and vegetables was observed among women $(p=0.01)$, among those with higher levels of education and white ethnicity, and higher consumption of beans was found among men $(p=0.04)$. The consumption of meat with excess fat was higher among men 
$(p<0.01)$, and the regular consumption of sweets $(p=0.02)$ and replacement of meals with snacks $(p=0.01)$ were higher among women, both directly associated with education. Conclusion: Significant differences were observed in food consumption between men and women living in Cuiabá, Mato Grosso. Education and ethnicity/skin color were variables that were associated with most of the food consumption markers.

Descriptors: Food Consumption; Epidemiological Surveys; Public Health Surveillance.

\section{RESUMEN}

Objetivo: Analizar la frecuencia del consumo de alimentos marcadores de dieta saludable y no saludable entre hombres y mujeres adultos y los factores demográficos y socioeconómicos asociados. Métodos: Estudio de corte transversal, de base poblacional con datos del Sistema de Vigilancia de Factores de Riesgo y Protección para Enfermedades Crónicas no Transmisibles a través de encuesta telefónica (VIGITEL). Fueron incluidos 1.005 adultos (400 hombres e 605 mujeres), entre 20 y 59 años que vivían en Cuiabá, Mato Grosso, entrevistados entre febrero y diciembre de 2014. Las variables del estudio fueron el consumo de alimentos marcadores de dieta saludable y no saludable y las variables demográficas y socioeconómicas. Para el análisis de los datos se utilizó el análisis bivariado a través de la prueba Chi-cuadrado (Rao-Scott) y la prueba de tendencia linear. Resultados: La media de edad de los participantes fue de 37,9 años para las mujeres y de 36,3 años para los hombres de los cuales el 50,9\% $(n=605)$ eran mujeres, el 60,0\% $(n=455)$ eran adultos jóvenes entre 20 y 39 años y de razal color negro o parda $(69,7 \% ; n=569)$. Fue observado mayor consumo regular de frutas y hortalizas entre las mujeres $(p=0,01)$ con más escolaridad y de la raza/color blanco y más consumo de frijoles entre los hombres $(p=0,04)$. El consumo de carnes con mucha grasa fue mayor entre los hombres $(p<0,01)$ así como el consumo regular de dulces $(p=0,02)$ y la sustitución de las comidas principales por meriendas fue mayor entre las mujeres ( $p=0,01)$, los dos directamente asociados con la escolaridad. Conclusión: Se verificaron diferencias importantes en el consumo de alimentos entre los hombres y mujeres residentes de Cuiabá, Mato Grosso. La escolaridad y la raza/color fueron las variables que se asociaron con la mayoría de los marcadores de consumo alimentario.

Descriptores: Consumo de Alimentos; Encuestas Epidemiológicas; Vigilancia en Salud Pública.

\section{INTRODUÇÃO}

Mudanças significativas têm ocorrido nos hábitos alimentares da população mundial e na qualidade da dieta, com aumento do consumo de alimentos ultraprocessados e industrializados, com elevada densidade energética, ricos em açúcares refinados, sódio e gorduras, especialmente gorduras trans e gorduras saturadas, e pobres em fibras; em detrimento do consumo de alimentos in natura, como frutas, verduras, legumes e cereais integrais ${ }^{(1,2)}$. No Brasil, houve uma diminuição da disponibilidade domiciliar de alimentos básicos tradicionais da dieta, como o arroz e o feijão ${ }^{(3)}$.

Dietas pouco saudáveis, juntamente com inatividade física, consumo nocivo de bebidas alcoólicas e tabagismo são os principais fatores de risco para o desenvolvimento de doenças crônicas não transmissíveis (DCNT) $)^{(4)}$, como as doenças cardiovasculares, cânceres, diabetes e doenças respiratórias crônicas ${ }^{(5,6)}$.

A proporção de adultos com excesso de peso tem aumentando de forma progressiva e, no Brasil, esse aumento tem sido verificado em todos os inquéritos nacionais realizados desde a década de $1970^{(5)}$. Nesse contexto, a população da cidade de Cuiabá, capital do estado de Mato Grosso - Brasil, tem se destacado pela elevada prevalência de indivíduos adultos com excesso de peso, sobretudo com obesidade, em ambos os $\operatorname{sexos}^{(7)}$.

Devido à relevância das DCNT no perfil epidemiológico da população brasileira e ao fato de seus principais fatores de risco serem passíveis de prevenção, o Ministério da Saúde implantou, em 2006, a Vigilância de fatores de risco e proteção para doenças crônicas por inquérito telefônico (VIGITEL) ${ }^{(8)}$, dentro do Sistema de vigilância de fatores de risco para doenças crônicas não transmissíveis (DCNT), que reúne características potenciais de simplicidade, baixo custo e agilidade ${ }^{(9)}$. O monitoramento constante dos indicadores de risco e proteção para DCNT fornece subsídios para as políticas de promoção da saúde no Brasil, inclusive para o incentivo a uma alimentação mais saudável, haja vista uma parte do questionário do VIGITEL abordar a frequência de consumo de alimentos considerados marcadores de dieta saudável e não saudável.

Assim sendo, o objetivo deste estudo é analisar a frequência de consumo de alimentos marcadores de dieta saudável e não saudável entre homens e mulheres adultos e os fatores demográficos e socioeconômicos associados.

\section{MÉTODOS}

Estudo epidemiológico de corte transversal, de base populacional, com dados individuais de adultos residentes em Cuiabá, capital do Estado de Mato Grosso - Brasil, entrevistados pelo inquérito telefônico VIGITEL.

Os procedimentos de amostragem empregados pelo VIGITEL visam obter amostras probabilísticas da população de adultos ( $\geq 18$ anos de idade) que residem em domicílios servidos por, ao menos, uma linha telefônica fixa, em cada uma das capitais dos 26 estados brasileiros e no Distrito Federal. O sistema estabelece um tamanho amostral mínimo de aproximadamente 1.500 
indivíduos em cada cidade para estimar, com coeficiente de confiança de $95 \%$ e erro máximo de cerca de 3\%, a frequência de qualquer fator de risco na população adulta ${ }^{(8)}$.

Os critérios de inclusão do estudo foram: idade entre 20 a 59 anos, ser residente em Cuiabá e ter sido entrevistado(a) pelo VIGITEL no período de fevereiro a dezembro de 2014. Adotou-se como critério de exclusão as mulheres que responderam "sim" ou "não sabe" à questão sobre estar grávida no momento da entrevista.

No ano de 2014, foram realizadas pelo VIGITEL, em Cuiabá, 1.509 entrevistas telefônicas ( 575 homens e 934 mulheres ${ }^{(8)}$. Desse total, foram elegíveis para a amostra do estudo 1.019 indivíduos, com idade entre 20 e 59 anos (400 homens e 619 mulheres), porém foram excluídas 14 mulheres, pois 9 disseram não saber e 5 mulheres confirmaram que estavam grávidas no momento da entrevista. Dessa forma, a amostra do presente estudo foi constituída por 1.005 indivíduos, de 20 a 59 anos (400 homens e 605 mulheres).

As entrevistas telefônicas do VIGITEL 2014 foram realizadas por uma empresa especializada através de computadores, com as perguntas lidas diretamente na tela de um monitor de vídeo e as respostas registradas direta e imediatamente em meio eletrônico ${ }^{(8)}$.

Para este estudo, utilizou-se apenas uma parte das perguntas do questionário VIGITEL 2014, que abordam características demográficas e socioeconômicas dos indivíduos e características do consumo alimentar.

As variáveis socioeconômicas e demográficas investigadas foram: sexo, idade, escolaridade, situação conjugal e raça/cor. A idade referida em anos completos foi agrupada em faixas etárias (20 a 39; 40 a 59 anos) e a escolaridade referida em anos completos de estudo, sendo agrupada em 3 categorias: 0 a $8 ; 9$ a 11 ou $\geq 12$ anos. O estado conjugal foi coletado conforme as opções solteiro(a), casado(a) legalmente, em união estável há mais de 6 meses, viúvo(a), separado(a) ou divorciado(a), e a variável foi posteriormente dicotomizada em "com companheiro(a)" ou "sem companheiro(a)" [solteiro(a), viúvo(a) ou separado(a)/divorciado(a)]. A variável raça/cor foi coletada como branca, preta, parda, amarela (ascendência oriental) e vermelha (ascendência indígena), e posteriormente agrupada em "branca" e "preta ou parda", sendo excluídos da análise os indivíduos que referiram raça/cor amarela $(\mathrm{n}=43)$ ou vermelha $(\mathrm{n}=13)$, devido à baixa frequência.

Avaliou-se o consumo de alimentos pelo uso de marcadores de dieta saudável e não saudável de acordo com a nomenclatura utilizada no VIGITEL ${ }^{(8)}$. São considerados marcadores de dieta saudável o consumo regular de frutas e hortaliças (5 ou mais dias/semana), consumo recomendado de frutas e hortaliças (consumo de 5 porções em pelo menos 5 dias/semana) e consumo regular de feijão (5 ou mais dias/semana).

Dentre os marcadores de dieta não saudável, está o consumo de carnes com excesso de gordura (carne vermelha com gordura aparente e frango com pele); o consumo de leite com teor integral de gordura (consideradas as respostas "leite integral", "os dois tipos" ou "não sabe"); o consumo regular de doces, tais como sorvetes, chocolates, bolos, biscoitos ou doces (5 ou mais dias/semana); o consumo regular de refrigerantes ou refresco/suco artificial (5 ou mais dias/semana); o hábito de substituir as refeições principais (almoço e jantar) por lanches, tais como sanduíches, salgados, pizza ou outros lanches (7 ou mais vezes/ semana); e o consumo autorreferido de alta ou muito alta quantidade de sal (somando a comida preparada na hora e os alimentos industrializados).

Os fatores de ponderação que levam em consideração o caráter complexo da amostra foram utilizados para todas as análises. Os dados foram analisados pelo software Stata ${ }^{(10)}$, versão 13, utilizando-se o comando survey que aplica os fatores de ponderação. Foram estimadas frequências relativas das variáveis, com seus respectivos intervalos de confiança de $95 \%$, e realizada análise bivariada por meio do teste de qui-quadrado (Rao-Scott) entre as variáveis de consumo alimentar e as variáveis socioeconômicas e demográficas, para homens e mulheres. Quando indicado, realizou-se o teste de tendência linear. Foram considerados estatisticamente significativos os resultados com $\mathrm{p}<0,05$.

O presente estudo é derivado do projeto intitulado "Associação entre marcadores de consumo alimentar e excesso de peso em adultos de Cuiabá-MT, VIGITEL 2014", aprovado pelo Comitê de Ética em Pesquisa do Hospital Universitário Júlio Muller - Universidade Federal de Mato Grosso (CEP HUJM/UFMT - Parecer 1.443.713, de 09/03/2016). O projeto VIGITEL foi aprovado pela Comissão Nacional de Ética em Pesquisa (CONEP-MS - Parecer 355.590, de 26/6/2013).

\section{RESULTADOS}

A média de idade dos participantes foi de 37,9 anos (IC95\% 36,9; 39,0) para as mulheres e 36,3 anos (IC95\% 35,0; 37,6) para os homens. Dentre os entrevistados, $50,9 \%(\mathrm{n}=605)$ eram mulheres, e a maioria eram adultos jovens de 20 a 39 anos $(60,0 \% ; \mathrm{n}=455)$, de raça/cor preta ou parda $(69,7 \% ; \mathrm{n}=569)$ e predominaram indivíduos que disseram viver com companheiro(a) $(52,4 \% ; \mathrm{n}=559)$. A maioria dos indivíduos apresentou 9 ou mais anos completos de estudo $(70,9 \% ; \mathrm{n}=803)$, sendo que $37,0 \%$ $(\mathrm{n}=276)$ das mulheres e 29,6\% $(\mathrm{n}=148)$ dos homens apresentaram níveis de escolaridade de 12 ou mais anos completos de estudo.

Na Tabela I são apresentadas as frequências de consumo de alimentos marcadores de dieta saudável e não saudável segundo a variável sexo. Houve diferença significativa no consumo regular de feijão e de carnes com excesso de gordura, maior entre 
os homens; e no consumo regular de frutas e hortaliças, no consumo regular de doces e no hábito de substituir as refeições principais por lanches, maior entre as mulheres.

Nas Tabelas II a III são apresentadas as frequências de consumo de alimentos marcadores de dieta segundo variáveis demográficas e socioeconômicas estratificadas por sexo. Foram analisadas somente as variáveis marcadoras de consumo alimentar que tiveram diferença significativa entre os sexos $(\mathrm{p}<0,05)$, conforme visto na Tabela I.

Tabela I - Frequência de consumo de alimentos e respectivo intervalo de confiança de 95\% (IC 95\%) de homens e mulheres de 20 a 59 anos. Cuiabá, Mato Grosso, 2014.

\begin{tabular}{lccccccc}
\hline \multirow{2}{*}{\multicolumn{1}{c}{ Marcador de consumo alimentar }} & \multicolumn{2}{c}{ Total } & \multicolumn{2}{c}{ Masculino } & \multicolumn{2}{c}{ Feminino } & \multirow{2}{*}{ p-valor** } \\
\cline { 2 - 7 } & $\mathbf{\%}^{* *}$ & IC 95\% & \%* $^{*}$ & IC 95\% & \%* $^{*}$ & IC 95\% & \\
\hline Consumo regular de frutas e hortaliças & 30,7 & 27,$4 ; 34,3$ & 26,0 & 20,$9 ; 31,7$ & 35,2 & 30,$8 ; 39,9$ & 0,01 \\
Consumo recomendado de frutas e hortaliças & 18,8 & 16,$0 ; 21,9$ & 15,8 & 11,$8 ; 20,9$ & 21,7 & 18,$1 ; 25,8$ & 0,06 \\
Consumo regular de feijão & 77,2 & 73,$9 ; 80,2$ & 80,6 & 75,$5 ; 84,8$ & 73,9 & 69,$4 ; 77,9$ & 0,04 \\
Consumo de carnes com excesso de gordura & 46,6 & 42,$8 ; 50,6$ & 56,4 & 50,$4 ; 62,3$ & 37,3 & 32,$6 ; 42,2$ & $<0,01$ \\
Consumo de leite com teor integral de gordura & 51,0 & 47,$1 ; 54,9$ & 54,9 & 48,$8 ; 60,9$ & 47,3 & 42,$5 ; 52,2$ & 0,06 \\
Consumo de alimentos doces regularmente & 16,7 & 14,$1 ; 19,7$ & 13,0 & 9,$5 ; 17,7$ & 20,3 & 16,$7 ; 24,4$ & 0,02 \\
Consumo de refrigerante regularmente & 29,3 & 25,$7 ; 33,1$ & 32,1 & 26,$6 ; 38,1$ & 26,6 & 22,$3 ; 31,3$ & 0,14 \\
Substituição das refeições principais por lanches & 12,3 & 10,$1 ; 14,8$ & 9,0 & 6,$2 ; 12,7$ & 15,4 & 12,$3 ; 19,2$ & 0,01 \\
Consumo de teor alto ou muito alto de sal & 18,0 & 15,$2 ; 21,2$ & 19,5 & 15,$2 ; 24,6$ & 16,7 & 13,$2 ; 20,7$ & 0,36 \\
\hline **
\end{tabular}

*Percentual da amostra ponderada; ${ }^{* *}$ Teste de Qui-quadrado (Rao-Scott).

O consumo regular de frutas e hortaliças entre as mulheres foi associado ao maior nível de escolaridade e à raça/cor branca. Entre os homens, a frequência do consumo regular de frutas e hortaliças aumentou em média 9,3 pontos percentuais conforme $o$ aumento da escolaridade $(\mathrm{p}=0,01)$ e também esteve associado à raça/cor branca. Já para o consumo regular de feijão não houve associação significativa com as variáveis demográficas e socioeconômicas para ambos os sexos (Tabela II).

O autorrelato de consumo de carnes com excesso de gordura entre os homens não apresentou associação com variáveis demográficas e socioeconômicas. No entanto, entre as mulheres, o consumo de carnes com excesso de gordura foi associado à menor faixa etária ( $20 \mathrm{a} 39$ anos) e à raça/cor preta ou parda (Tabela IV). A frequência do consumo regular de doces entre as mulheres aumentou em média 10,4 pontos percentuais conforme o aumento da escolaridade $(\mathrm{p}<0,01)$ e predominou na faixa etária mais jovem (20 a 39 anos). Para ambos os sexos, o consumo regular de doces foi maior na raça/cor branca $(\mathrm{p}<0,05)$ (Tabela IV).

O hábito de substituir as refeições principais por lanches 7 ou mais vezes por semana foi maior entre as mulheres, com aumento proporcional à melhoria da escolaridade (em média, 8,2 pontos percentuais; $\mathrm{p}<0,01$ ) e em mulheres de raça/cor branca. Entre os homens, o fato de não ter companheiro (a) foi associado ao hábito de substituição das refeições principais por lanches (Tabela III). 


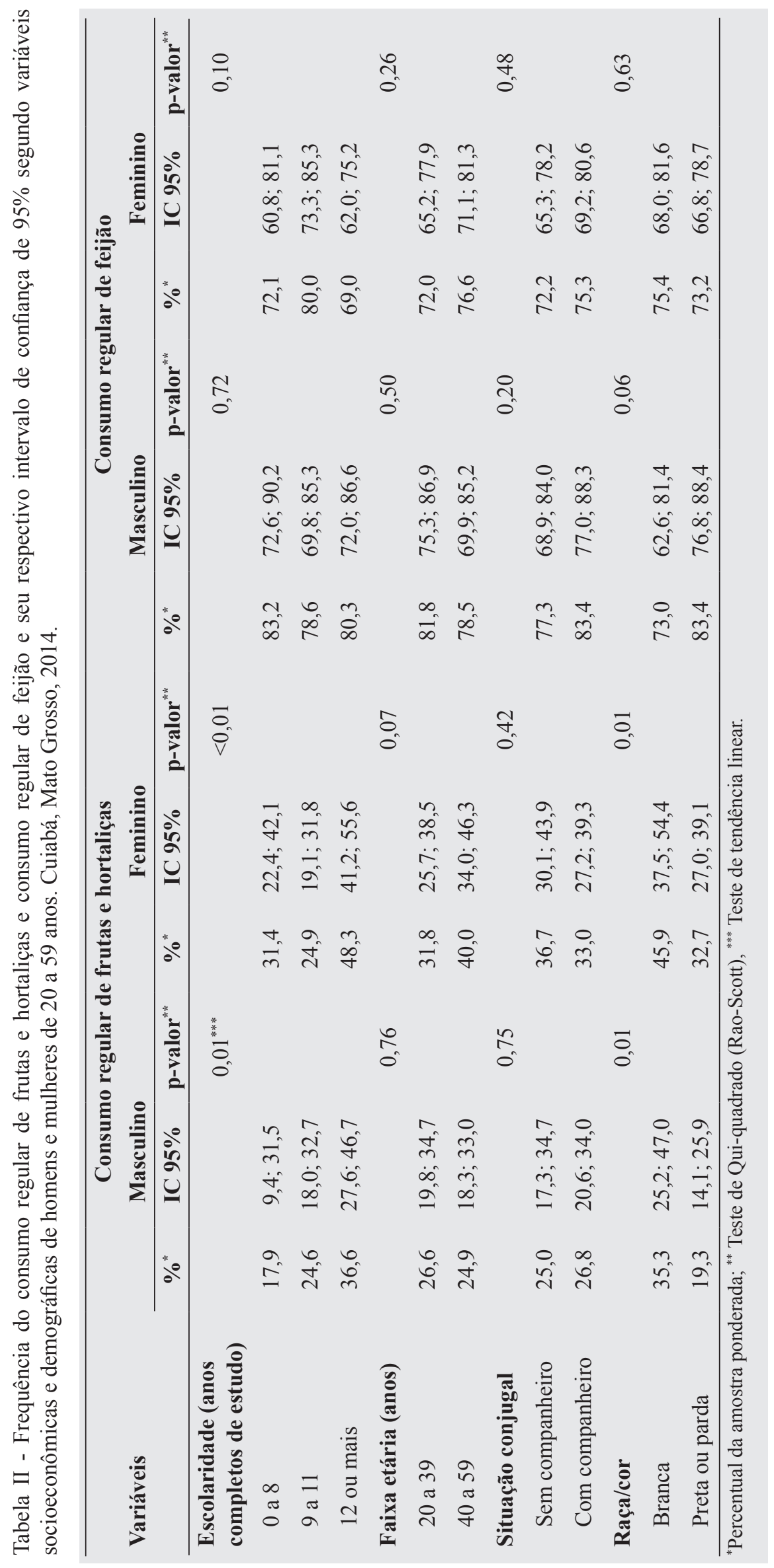









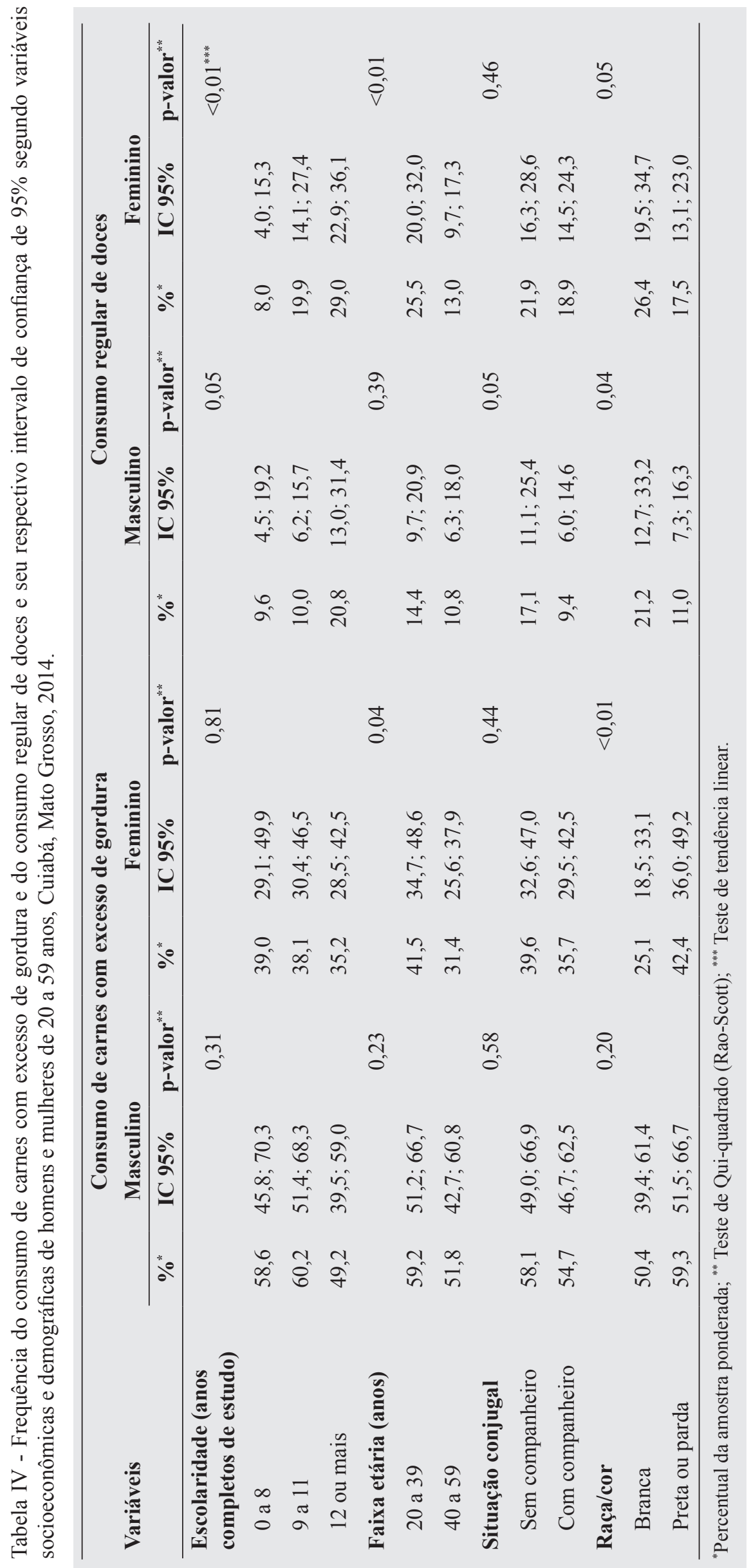




\section{DISCUSSÃO}

Os resultados do presente estudo revelaram diferenças no consumo de alimentos marcadores de padrões saudáveis e não saudáveis de alimentação entre homens e mulheres. O consumo de alimentos marcadores de dieta saudável foi caracterizado pelo maior consumo regular de frutas e hortaliças entre as mulheres e pelo consumo regular de feijão maior entre os homens. Com relação aos alimentos marcadores de dieta não saudável, observou-se o consumo mais frequente de carnes com excesso de gordura entre os homens e o consumo regular de doces e a substituição das refeições principais por lanches pelas mulheres.

Dentre as práticas alimentares saudáveis, a $\mathrm{OMS}^{(11)}$ recomenda o consumo diário mínimo de 5 porções de frutas e hortaliças, que corresponde à 400 gramas diários desses alimentos. As frutas, legumes e verduras são ricos em vitaminas, minerais e fibras, possuindo baixo teor energético ${ }^{(12)}$ em geral e seu consumo adequado auxilia na prevenção de DCNT e do ganho de peso excessivo $^{(13)}$, que é fator de risco para essas doenças ${ }^{(12,13)}$.

Somente $18,8 \%$ da população de estudo relatou consumo recomendado de frutas e hortaliças, sem diferença entre os sexos. Esses dados são condizentes com outros estudos realizados no mundo ${ }^{(14,15)}$ e em outras regiões do Brasil ${ }^{(16,17)}$, em que se verificou que elevada parcela da população consome frutas e hortaliças aquém do recomendado. Na análise do consumo regular de frutas e hortaliças, verificou-se que 30,7\% dos indivíduos disseram consumir frutas e hortaliças em 5 ou mais dias da semana. Essa proporção, apesar de representar pouco mais de 1/3 da população, foi maior que a encontrada em estudos realizados em outras regiões do Brasil ${ }^{(16-18)}$.

Em um estudo transversal realizado em Pelotas-RS(18) , com uma amostra de 972 indivíduos, de 20 a 69 anos, os autores verificaram que somente $1 / 5$ dos entrevistados $(20,9 \%)$ relatou consumo regular de frutas e hortaliças. Essa frequência foi substancialmente maior para mulheres $(26,9 \%)$ do que para homens $(12,9 \%)$, diferença também encontrada no presente estudo. $\mathrm{O}$ maior consumo de frutas e hortaliças por pessoas do sexo feminino parece estar relacionado à melhor percepção por parte dessas dos benefícios do consumo para a saúde ou para prevenção/controle de doenças ${ }^{(19)}$ e à associação com uma alimentação mais saudável ${ }^{(20)}$.

Adicionalmente, verificou-se, neste estudo, que o consumo regular de frutas e hortaliças foi mais frequente entre mulheres com 12 anos ou mais de estudo, e aumentou proporcionalmente à medida que melhorou a escolaridade entre os homens. Para ambos os sexos, maior frequência de consumo regular de frutas e hortaliças foi observada na raça/cor branca. Em um estudo transversal ${ }^{(15)}$, realizado no Canadá com 93.719 indivíduos, de 18 a 69 anos de idade, em que foi investigada a frequência de consumo diário de frutas e hortaliças e os fatores associados, os autores também verificaram que grupos de indivíduos com menor padrão socioeconômico (baixa renda e escolaridade) consumiam frutas e hortaliças menos frequentemente que aqueles com melhor renda e maior escolaridade ${ }^{(15)}$. Em Pelotas, Rio Grande do Sul ${ }^{(18)}$, verificaram maior consumo regular de frutas e hortaliças entre indivíduos de melhor nível socioeconômico (A e B), sem diferença entre raça/cor.

A variável raça/cor pode ser entendida como um marcador de desigualdade social ${ }^{(21)}$ e, no Brasil, apesar dos avanços na distribuição de renda, indivíduos da raça/cor preta ou parda figuram em maior proporção nos estratos de menor renda ${ }^{(22)}$. O consumo de frutas e hortaliças é influenciado diretamente pelo seu preço e pelo poder aquisitivo, de forma que, em geral, indivíduos de maior estrato de renda gastam mais com alimentos e compram mais frutas e hortaliças ${ }^{(19,23)}$.

Elevado consumo regular de feijão foi observado entre indivíduos de ambos os sexos, porém maior entre os homens, corroborando com outros estudos nacionais ${ }^{(24,25)}$. Não foram verificadas diferenças significantes no consumo de feijão segundo as variáveis demográficas e socioeconômicas para ambos os sexos, o que sugere que esse consumo esteja atrelado ao hábito alimentar brasileiro. O consumo de feijão, em combinação com arroz, é parte da dieta tradicional brasileira, e essa combinação é considerada nutricionalmente adequada pela complementação de aminoácidos essenciais ${ }^{(12)}$. Entretanto, os resultados da Pesquisa de Orçamentos Familiares (POF) ${ }^{(3)}$, sobre a disponibilidade domiciliar de alimentos, demonstraram que houve uma redução da quantidade média per capita adquirida de feijão, que passou de 12,4 kg em 2002-2003 para 9,1 kg em 2008-2009, o que representa uma redução de $26,4 \%$ na aquisição desse alimento, embora seu consumo possa ser ainda maior, uma vez que os dados não incluem o consumo extradomiciliar. Entretanto, dados recentes do VIGITEL apontam que o consumo de feijão se manteve estável na última década e, entre as capitais brasileiras, Cuiabá é a quarta com maior consumo do feijão no país ${ }^{(8)}$.

Entre os marcadores de dieta não saudável, observou-se que 46,6\% afirmaram consumir carnes com excesso de gordura e 51,0\% disseram consumir leite com teor integral de gordura. Alimentos de origem animal, que incluem as carnes, aves, peixes, ovos, leite e derivados, são importantes fontes de proteínas de alto valor biológico, ferro e vitaminas ${ }^{(12)}$. Tais alimentos, entretanto, também são ricos em gordura saturada, que tem sido relacionada a maior risco de desenvolvimento de doenças cardiovasculares $^{(26)}$. O Guia Alimentar para a População Brasileira ${ }^{(12)}$ recomenda que os adultos devem, sempre que possível, escolher leite e derivados com menores quantidades de gorduras, e devem preferir as carnes magras, retirando toda a gordura aparente antes da preparação.

O maior consumo de carnes entre os homens tem sido documentado em outros estudos nacionais ${ }^{(21,25,27,28)}$. Neste estudo, entre os homens não houve diferenças significativas no consumo de carnes com excesso de gordura segundo as variáveis demográficas e socioeconômicas. Porém maior consumo foi verificado entre mulheres na faixa etária mais jovem (20 a 39 
anos) e de raça/cor preta ou parda. Dados de estudo transversal(21) com 45.448 adultos das capitais brasileiras e Distrito Federal entrevistados pelo VIGITEL em 2012, também apontaram que, entre as mulheres, aquelas que se declararam pretas ou pardas consumiam mais carne com excesso de gordura em relação às brancas. Em um estudo de base populacional ${ }^{(28)}$ realizado na zona $^{2}$ urbana de Pelotas, Rio Grande do Sul, em 2010, com 2.732 adultos com 20 anos ou mais, que objetivou descrever a frequência do consumo de carnes e o hábito de consumi-las com excesso de gordura, os autores verificaram que as carnes com excesso de gordura foram consumidas por $52,3 \%$ dos adultos, principalmente homens $(66,7 \%)$, e por pessoas com idade entre 30 e 39 anos, de menor escolaridade e nível econômico (classes D e E). Os dados estratificados por sexo, porém, não foram apresentados, impedindo a comparação com os achados do presente estudo. Dados da Pesquisa Nacional de Saúde de 2013 apontam que o estado do Mato Grosso apresenta a segunda maior prevalência de consumo de carne com excesso de gordura do país, mostrando que é um hábito regional tradicional ${ }^{(29)}$.

$\mathrm{A} \mathrm{OMS}^{(2)}$ recomenda que a ingestão de açúcares livres deve ser reduzida para menos de $10 \%$ do consumo total de energia diário, que é equivalente a 50 g para uma pessoa eutrófica, que consome cerca de 2000 calorias por dia. Açúcares livres são todos os açúcares adicionados aos alimentos ou bebidas e também aqueles naturalmente presentes no mel, xaropes, sucos de frutas naturais e concentrados. Sua ingestão pode ser reduzida limitando o consumo de bebidas adoçadas com açúcar, refrigerantes e doces em geral $^{(2)}$. Observou-se na população deste estudo elevado consumo regular de doces e refrigerantes, fontes de açúcares livres. Dentre os indivíduos de ambos os sexos, 16,7\% disseram consumir regularmente doces e 29,3\% relataram consumo regular de refrigerantes. O consumo de doces, tais como sorvetes, chocolates, bolos, biscoitos ou doces, em 5 ou mais dias da semana, foi significativamente maior entre mulheres $(20,3 \%)$, associado diretamente ao aumento da escolaridade, mais frequente entre mulheres mais jovens e da raça/cor branca.

Em um inquérito de base populacional ${ }^{(30)}$ realizado com 3.136 indivíduos, de 20 anos ou mais, residentes na zona urbana de Pelotas, utilizando um questionário pré-codificado sobre os 10 Passos da Alimentação Saudável, que é baseado nas recomendações do Guia Alimentar para a População Brasileira ${ }^{(12)}$, foi verificado que, entre as mulheres, a menor adesão ao passo 6 (consumo de doces, bolos, biscoitos e outros alimentos ricos em açúcar no máximo duas vezes por semana) foi observada naquelas de menor idade (20 a 39 anos) e de maior escolaridade, dados semelhantes aos resultados encontrados no presente estudo.

A diferença no consumo de doces entre homens e mulheres tem sido descrita em outros estudos ${ }^{(25,29,31,32)}$. Em um estudo de corte transversal $^{(32)}$ realizado com dados de 37.181 franceses, de idade $\geq 20$ anos, que preencheram questionários on-line, os autores investigaram a associação entre o gosto pelos sabores salgado e doce e as características sociodemográficas, psicológicas e de estilo de vida. No estudo, verificaram que o gosto pelo sal aumentou com a idade, especialmente nos homens, ao passo que o gosto pelo sabor doce diminuiu com a idade, principalmente entre as mulheres. Esse resultado corrobora com o maior consumo de doces entre mulheres de 20 a 39 anos, observado no presente estudo. Fumantes e bebedores de álcool tinham maior probabilidade de gosto pelo salgado ${ }^{(32)}$. Em geral, homens consomem maior quantidade de bebidas alcoólicas e fumam mais que as mulheres ${ }^{(31)}$, o que poderia, em parte, explicar seu menor consumo de doces. Além disso, os autores verificaram que o gosto por doces foi maior nas mulheres com "comer emocional”, que é a propensão a comer em excesso em relação aos estados de humor negativos ${ }^{(32)}$.

Diferentes hipóteses podem ser levantadas sobre a preferência por doces entre as mulheres de maior escolaridade. Além das características já citadas, relacionadas ao gosto pelo sabor doce entre mulheres, o estresse que a mulher está sujeita devido à sobrecarga de atividades/trabalho e o maior poder aquisitivo disponível para compra de elevada variedade de alimentos ${ }^{(23)}$ e, consequentemente, de produtos processados e ultraprocessados, que têm uma oferta cada vez maior, acompanhada de propagandas e promoções para estímulo à compra ${ }^{(33)}$, poderiam estar relacionados ao maior consumo de doces nessa população.

O hábito de substituir as refeições por lanches, como sanduíches, salgados, pizza ou outros lanches, sete ou mais vezes por semana, foi verificado em $12,3 \%$ da população estudada, sendo maior entre mulheres, com elevação da frequência proporcional ao aumento da escolaridade. Entre os homens, a maior frequência de substituição das refeições principais por lanches foi verificada naqueles que viviam sem companheira (solteiros, separados/divorciados e viúvos).

A substituição frequente do almoço e/ou jantar por lanches provavelmente decorre de fatores relacionados a mudanças no estilo de vida dos indivíduos, que tem ocorrido ao longo dos anos, com uma tendência de "conveniência e praticidade", com realização frequente de refeições fora de casa, motivadas principalmente pelo ritmo de vida nos centros urbanos, a inserção da mulher no mercado de trabalho e a falta de tempo para o preparo dos alimentos, além de perda das habilidades na culinária, o que estimula a demanda por produtos ultraprocessados, com maior teor de sal, gorduras e conservantes ${ }^{(33-35)}$, que aumentam o risco de ganho de peso excessivo e outras doenças crônicas ${ }^{(12,26,35)}$.

No Guia Alimentar para a População Brasileira 2014 ${ }^{(33)}$ foi adotada como regra de ouro a instrução de preferir sempre alimentos in natura, ou minimamente processados, e preparações culinárias a alimentos ultraprocessados. A recomendação é comer com regularidade e com atenção, procurando fazer refeições diárias em horários semelhantes e evitando "beliscar" nos intervalos entre as refeições, o que inclui a valorização do momento das refeições, comendo em ambientes apropriados, em companhia, além de compartilhar as atividades domésticas que antecedem ou sucedem o consumo das refeições. Essas orientações devem fazer parte das recomendações dos profissionais de saúde, buscando resgatar o preparo dos alimentos, a culinária local e o prazer de compartilhar a alimentação com familiares, amigos, colegas de trabalho ou escola ${ }^{(33)}$. 
O autorrelato de consumo de teor elevado ou muito elevado de sal nas preparações alimentares foi expressivo na população de estudo (18,0\%), sem diferença estatisticamente significativa entre os sexos. Em um estudo transversal ${ }^{(36)}$, utilizando dados da Pesquisa Nacional de Saúde (PNS) e do VIGITEL 2013, os autores verificaram que, no Brasil, a prevalência de adultos que referiram consumo elevado de sal foi de $14,2 \%$ (PNS) e de 15,0\% (VIGITEL) para o total das capitais, sendo maior entre indivíduos do sexo masculino.

Os autores ressaltam que os resultados do estudo não podem ser vistos como uma aproximação do real consumo de sal pela população adulta do país, já que dados do Inquérito Nacional de Alimentação da POF 2008-2009 indicavam ingestão inadequada e excessiva de sódio por $89,3 \%$ dos homens e $70 \%$ das mulheres do país ${ }^{(37)}$, e o consumo elevado de sal foi identificado apenas por $16,1 \%$ dos homens e $12,5 \%$ das mulheres participantes da PNS $^{(36)}$. A comparação demonstra que a capacidade da população de reconhecer seu consumo de sal permanece muito abaixo do ideal.

No presente estudo, as diferenças entre os métodos para avaliar a dieta e para definir e categorizar a frequência de consumo de alimentos prejudicam a comparação com outros estudos sobre consumo alimentar populacional. Além disso, todas as informações obtidas foram autorrelatadas, permitindo vieses por lapsos de memória ou mesmo por depoimentos tendenciosos na direção do desejável. Estudos de comparação de estimativas para o autorrelato de condições crônicas entre inquéritos domiciliares e telefônicos têm demonstrado, entretanto, que ambos fornecem resultados semelhantes ${ }^{(38,39)}$, inclusive para obtenção de marcadores nutricionais ${ }^{(40)}$.

A cobertura telefônica é um dos principais limitantes dos estudos feitos a partir de inquéritos telefônicos, uma vez que a cobertura dessa rede não é evidentemente universal ${ }^{(9)}$, podendo ser particularmente baixa em cidades economicamente menos desenvolvidas e nos estratos de menor nível socioeconômico ${ }^{(8)}$. O uso de pesos pós-estratificação, porém, permite igualar a composição sociodemográfica estimada para a população de adultos com telefone a partir da amostra VIGITEL em cada cidade à composição sociodemográfica que se estima para a população adulta total da mesma cidade e no mesmo ano de realização do levantamento ${ }^{(8,9)}$. Políticas públicas dirigidas à educação nutricional e ao incentivo à alimentação saudável são urgentes e necessárias.

\section{CONCLUSÃO}

No presente estudo, verificaram-se diferenças importantes no consumo de alimentos entre homens e mulheres residentes em Cuiabá, Mato Grosso, marcadas pelo maior consumo regular de frutas e hortaliças entre as mulheres e elevado consumo regular de feijão entre os homens. Homens autorrelataram um maior consumo de carnes com excesso de gordura, e as mulheres, maior consumo regular de doces e hábito de substituir as refeições principais por lanches. A escolaridade e a raça/cor foram variáveis que se associaram à maioria dos marcadores de consumo alimentar.

\section{REFERÊNCIAS}

1. Louzada MLC, Martins APB, Canella DS, Levy RB, Claro RM, Moubarac JC, et al. Alimentos ultraprocessados e perfil nutricional da dieta no Brasil. Rev Saude Publica. 2015;49:38.

2. World Health Organization. Media Centre. Healthy diet: Fact sheet no 394 [Internet]. Geneva: WHO; 2015 [acesso em 2017 Jan 28]. Disponível em: http://www.who.int/mediacentre/factsheets/fs394/en/

3. Instituto Brasileiro de Geografia e Estatística (BR). Pesquisa de orçamentos familiares (POF) 2008-2009: aquisição alimentar domiciliar per capita Brasil e Grandes Regiões. Rio de Janeiro: IBGE; 2010.

4. World Health Organization. Noncommunicable diseases country profiles 2011. Geneva: WHO; 2011.

5. Ministério da Saúde (BR), Secretaria de Vigilância em Saúde, Departamento de Análise de Situação de Saúde. Plano de ações estratégicas para o enfrentamento das doenças crônicas não transmissíveis (DCNT) no Brasil 2011-2022. Brasília: Ministério da Saúde; 2011. (Série B. Textos Básicos de Saúde).

6. Ministério da Saúde (BR), Secretaria de Vigilância em Saúde, Departamento de Análise de Situação de Saúde. VIGITEL Brasil 2013: vigilância de fatores de risco e proteção para doenças crônicas por inquérito telefônico. Brasília: Ministério da Saúde; 2014. (Série G. Estatística e Informação em Saúde).

7. Serra-Majem L, Bautista-Castaño I. Etiology of obesity: two "key issues" and other emerging factors. Nutr Hosp. 2013;28(Suppl 5):32-43.

8. Ministério da Saúde (BR), Secretaria de Vigilância em Saúde, Departamento de Análise de Situação de Saúde. VIGITEL Brasil 2014: vigilância de fatores de risco e proteção para doenças crônicas por inquérito telefônico. Brasília: Ministério da Saúde; 2015. (Série G. Estatística e Informação em Saúde). 
9. Gubert MB, Santos LMP, Moura EC. Estratégias de diagnóstico nutricional rápido em populações. In: Taddei JAAC, Lang RMF, Longo-Silva G, Toloni MHA, editores. Nutrição em Saúde Pública. Rio de Janeiro: Rubio; 2011. p. 151-64.

10. StataCorp. Stata statistical software: release 13. College Station: StataCorp LP; 2013.

11. World Health Organization. Diet, nutrition and the prevention of chronic diseases. Report of a Joint WHO/FAO Expert Consultation. Geneva: WHO; 2003.

12. Ministério da Saúde (BR), Secretaria de Atenção à Saúde, Departamento de Atenção Básica, Coordenação-Geral da Política de Alimentação e Nutrição. Guia alimentar para a população brasileira: promovendo a alimentação saudável. Brasília: Ministério da Saúde; 2006. (Série A. Normas e Manuais Técnicos).

13. Boeing H, Bechthold A, Bub A, Ellinger S, Haller D, Kroke A, et al. Critical review: vegetables and fruit in the prevention of chronic diseases. Eur J Nutr. 2012;51(6):637-63.

14. Hall JN, Moore S, Harper SB, Lynch JW. Global variability in fruit and vegetable consumption. Am J Prev Med. 2009;36(5):402-9.

15. Azagba S, Sharaf MF. Disparities in the frequency of fruit and vegetable consumption by socio-demographic and lifestyle characteristics in Canada. Nutr J. 2011;10:118.

16. Mondini L, Moraes SA, Freitas ICM, Gimeno SGA. Consumo de frutas e hortaliças por adultos em Ribeirão Preto, SP. Rev Saúde Pública. 2010;44(4):686-94.

17. Azevedo ECC, Diniz AS, Monteiro JS, Cabral PC. Padrão alimentar de risco para as doenças crônicas não transmissíveis e sua associação com a gordura corporal: uma revisão sistemática. Ciênc Saúde Coletiva. 2014;19(5):1447-58.

18. Neutzling MB, Rombaldi AJ, Azevedo MR, Hallal PC. Fatores associados ao consumo de frutas, legumes e verduras em adultos de uma cidade no Sul do Brasil. Cad Saúde Pública. 2009;25(11):2365-74.

19. Figueira TR, Lopes ACS, Modena CM. Barreiras e fatores promotores do consumo de frutas e hortaliças entre usuários do Programa Academia da Saúde. Rev Nutr. 2016;29(1):85-95.

20. Figueira TR, Lopes ACS, Modena CM. Avaliação do consumo de frutas e hortaliças entre famílias de usuários do Programa Academia da Saúde (PAS). Rev Bras Promoç Saúde. 2014;27(4):518-26.

21. Malta DC, Moura L, Bernal RTI. Diferenciais dos fatores de risco de Doenças Crônicas não Transmissíveis na perspectiva de raça/cor. Ciênc Saúde Coletiva. 2015;20(3):713-25.

22. Instituto de Pesquisa Econômica Aplicada (BR). Retrato das desigualdades de gênero e raça. $4^{\mathrm{a}}$ ed. Brasília: IPEA; 2011.

23. Pechey R, Monsivais P. Socioeconomic inequalities in the healthiness of food choices: Exploring the contributions of food expenditures. Prev Med. 2016;88:203-9.

24. Velásquez-Meléndez G, Mendes LL, Pessoa MC, Sardinha LMV, Yokota RTC, Bernal RTI, et al. Tendências da frequência do consumo de feijão por meio de inquérito telefônico nas capitais brasileiras, 2006 a 2009. Ciênc Saúde Coletiva. 2012;17(12):3363-70.

25. Souza AM, Pereira RA, Yokoo EM, Levy RB, Sichieri R. Alimentos mais consumidos no Brasil: Inquérito Nacional de Alimentação 2008-2009. Rev Saúde Pública. 2013;47(Supl 1):190S-9S.

26. Mozaffarian D, Micha R, Wallace S. Effects on coronary heart disease of increasing polyunsaturated fat in place of saturated fat: a systematic review and meta-analysis of randomized controlled trials. PLoS Med. 2010;7(3):e1000252.

27. Carvalho AM, César CLG, Fisberg RM, Marchioni DML. Excessive meat consumption in Brazil: diet quality and environmental impacts. Public Health Nutr. 2013;16(10):1893-9.

28. Schneider BC, Duro SMS, Assunção MCF. Consumo de carnes por adultos do sul do Brasil: um estudo de base populacional. Ciênc Saúde Coletiva. 2014;19(8):3583-92.

29. Claro RM, Santos MAS, Oliveira TP, Pereira CA, Szwarcwald CL, Malta DC. Consumo de alimentos não saudáveis relacionados a doenças crônicas não transmissíveis no Brasil: Pesquisa Nacional de Saúde, 2013. Epidemiol Serv Saúde. 2015;24(2):257-65.

30. Vinholes DB, Assunção MCF, Neutzling MB. Frequência de hábitos saudáveis de alimentação medidos a partir dos 10 Passos da Alimentação Saudável do Ministério da Saúde. Pelotas, Rio Grande do Sul, Brasil. Cad Saúde Pública. 2009;25(4):791-9. 
31. Malta DC, Campos MO, Oliveira MM, Iser BPM, Bernal RTI, Claro RM, et al. Prevalência de fatores de risco e proteção para doenças crônicas não transmissíveis em adultos residentes em capitais brasileiras, 2013. Epidemiol Serv Saúde. 2015;24(3):373-87.

32. Lampuré A, Schlich P, Deglaire A, Castetbon K, Péneau S, Hercberg S, et al. Sociodemographic, psychological, and lifestyle characteristics are associated with a liking for salty and sweet tastes in French adults. J Nutr. 2015 Mar;145(3):587-94.

33. Ministério da Saúde (BR), Secretaria de Atenção à Saúde, Departamento de Atenção Básica. Guia alimentar para a população brasileira. $2^{\mathrm{a}}$ ed. Brasília: Ministério da Saúde; 2014.

34. Bezerra IN, Cavalcante JB, Moreira TMV, Mota CC, Sicheiri R. Alimentação fora de casa e excesso de peso: uma análise dos mecanismos explicativos. Rev Bras Promoç Saúde. 2016;29(3):455-61.

35. Bezerra IN, Souza AM, Pereira RA, Sichieri R. Contribution of foods consumed away from home to energy intake in Brazilian urban areas: the 2008-9 Nationwide Dietary Survey. Br J Nutr. 2013;109(7):1276-83.

36. Oliveira MM, Malta DC, Santos MAS, Oliveira TP, Nilson EAF, Claro RM. Consumo elevado de sal autorreferido em adultos: dados da Pesquisa Nacional de Saúde, 2013. Epidemiol Serv Saúde. 2015;24(2):249-56.

37. Araujo MC, Bezerra IN, Barbosa FS, Junger WL, Yokoo EM, Pereira RA, et al. Consumo de macronutrientes e ingestão inadequada de micronutrientes em adultos. Rev Saúde Pública. 2013;47(Supl 1):177S-89S.

38. Francisco PMSB, Barros MBA, Segri NJ, Alves MCGP, Cesar CLG, Malta DA. Comparação de estimativas para o auto-relato de condições crônicas entre inquérito domiciliar e telefônico - Campinas (SP), Brasil. Rev Bras Epidemiol. 2011;14(Supl 1):5-15.

39. Francisco PMSB, Barros MBA, Segri NJ, Alves MCGP. Comparação de estimativas de inquéritos de base populacional. Rev Saúde Pública. 2013;47(1):60-8.

40. Monteiro CA, Moura EC, Jaime PC, Claro RM. Validade de indicadores do consumo de alimentos e bebidas obtidos por inquérito telefônico. Rev Saúde Pública. 2008;42(4):582-9.

\section{Endereço para correspondência:}

Gabriela Dalcin Durante

Universidade Federal de Mato Grosso - UFMT

Campus Cuiabá, Bloco CCBS III, Nesan - Grupo de Pesquisa Epidemiologia em Saúde e Nutrição

Av. Fernando Corrêa da Costa, 2367

Bairro: Boa Esperança

CEP: 78060-900 - Cuiabá - MT - Brasil

E-mail: gabrielad.durante@gmail.com 